\title{
Antenatal Management of Second Trimester Oligohydramnios and Evolving Abnormal Ultrasound Findings: Case Report
}

\author{
Daniel Roshan, MD, FACOG, FACS*, Ellen Kolman, PA-C, Shira Plonka, MS CGC and Ilan \\ Timor, MD, FACOG
}

Department of Obstetrics and Gynecology, New York University School of Medicine, New York, USA

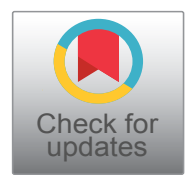

*Corresponding author: Daniel Roshan, MD, FACOG, FACS, Department of Obstetrics and Gynecology, New York University School of Medicine, 213 Madison Ave \#1A, New York, NY 10016, USA, Tel: (212)-725-0123

\begin{abstract}
Appropriate patient selection for prenatal intervention in the setting of abnormal antenatal ultrasound findings remains challenging. This case illustrates the course of a complicated pregnancy in a 35-year-old gravida 1 para 0 who presented to our Maternal-Fetal-Medicine clinic at 24.2 weeks gestation with fetal ascites, scalp edema, and oligohydramnios. Her first trimester ultrasound and genetic screening was normal. The patient was counseled that the observed findings carried a high chance for fetal demise or postnatal birth defects and complications. The patient chose to continue the pregnancy and opted for any necessary fetal interventions. Throughout the course of her pregnancy, multiple therapeutic interventions were performed in order to maintain a stable fetal environment, including intrauterine paracentesis, transabdominal amnioinfusion, vesicocentesis, and placement of a peritoneal amniotic shunt. The purpose of this case report is to investigate the role of fetal intervention in second trimester abnormal ultrasound findings and improve the quality of future prenatal consultation and therapy.
\end{abstract}

\section{Keywords}

Fetal intervention, Invasive fetal therapy, Oligohydramnios management, Intrauterine paracentesis, Transabdominal amnioinfusion, Peritoneal amniotic shunt, Case report

\section{Introduction}

Throughout this case, intrauterine fluid was manipulated through multiple procedures due to evolving ominous ultrasound findings. An intrauterine paracentesis of ascites fluid was initially performed to decompress the fetal abdomen, alongside an amnioinfusion to correct oligohydramnios. With all normal prenatal screen- ing and an unclear etiology, the leading initial hypothesis involved a possible intestinal perforation leaking amniotic fluid into the abdominal cavity. With gradually declining amniotic fluid index (AFI), a second amnioinfusion was performed at 26.5 weeks, and a peritoneal amniotic shunt was placed using a double pigtail catheter to bypass the suspected perforation. AFI was closely monitored and appeared to stabilize. At 28.2 weeks, AFI began to decline, and a distended bladder with a distinct keyhole appearance was seen on ultrasound, consistent with a fetal lower urinary tract obstruction (LUTO). Fetal kidneys and bladder appeared increasingly pronounced with bilateral hydronephrosis and bladder distension visualized on ultrasound. At 28.5 weeks, a third amnioinfusion along with a vesicocintesis was performed. Close monitoring revealed gradually worsening hydronephrosis but stable low-normal AFI over the upcoming weeks. At 34.5 weeks, AFI abruptly dropped. Poor profusion to right fetal kidney, and a sudden change in fetal urinary output led to an emergent cesarean section due to concern for pending renal failure.

\section{Case Report}

The patient is a 35-year-old gravida 1 para 0 who presented for an urgent consult to our Maternal Fetal Medicine group at 24.2 weeks gestation. Routine ultrasound with prior $\mathrm{OB}$ revealed sudden onset of fetal ascites and scalp edema in the absence of any prior pregnancy abnormalities. Repeat ultrasound confirmed findings (Figure 1 and Figure 2). Fetal echo was also performed at 24.2 weeks, and cardiac function was nor- 


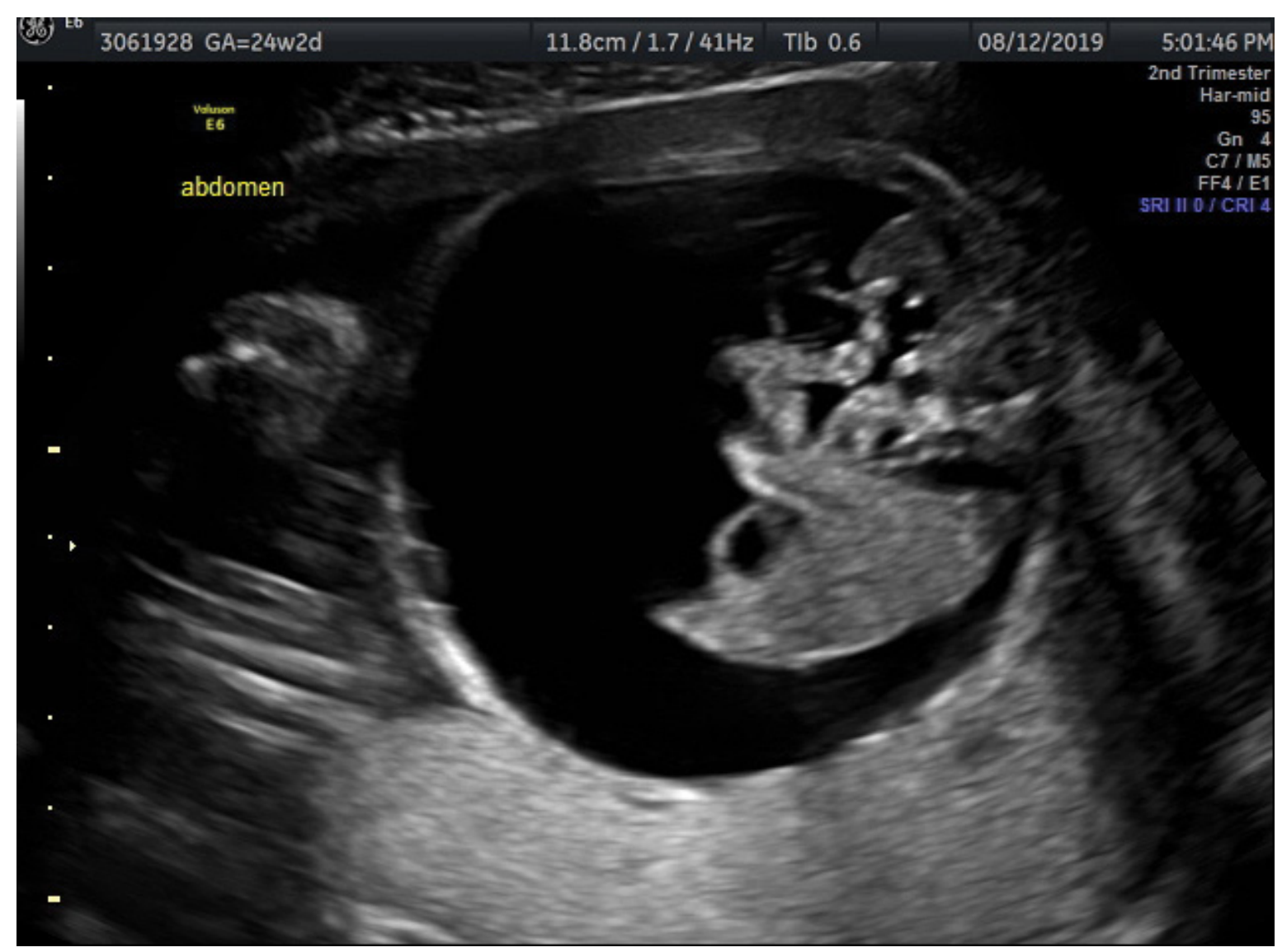

Figure 1: Fetal ascites.

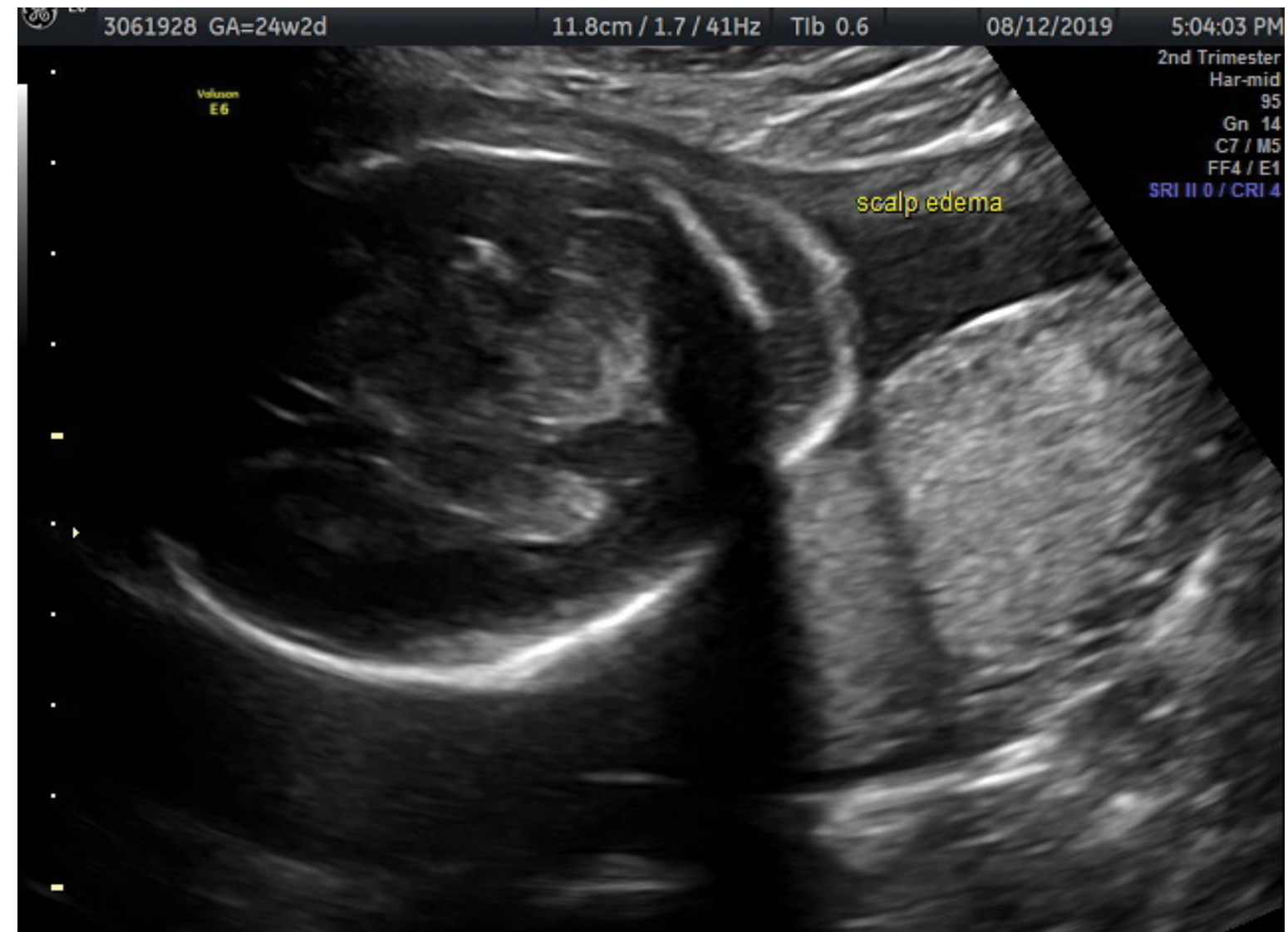

Figure 2: Fetal scalp edema. 


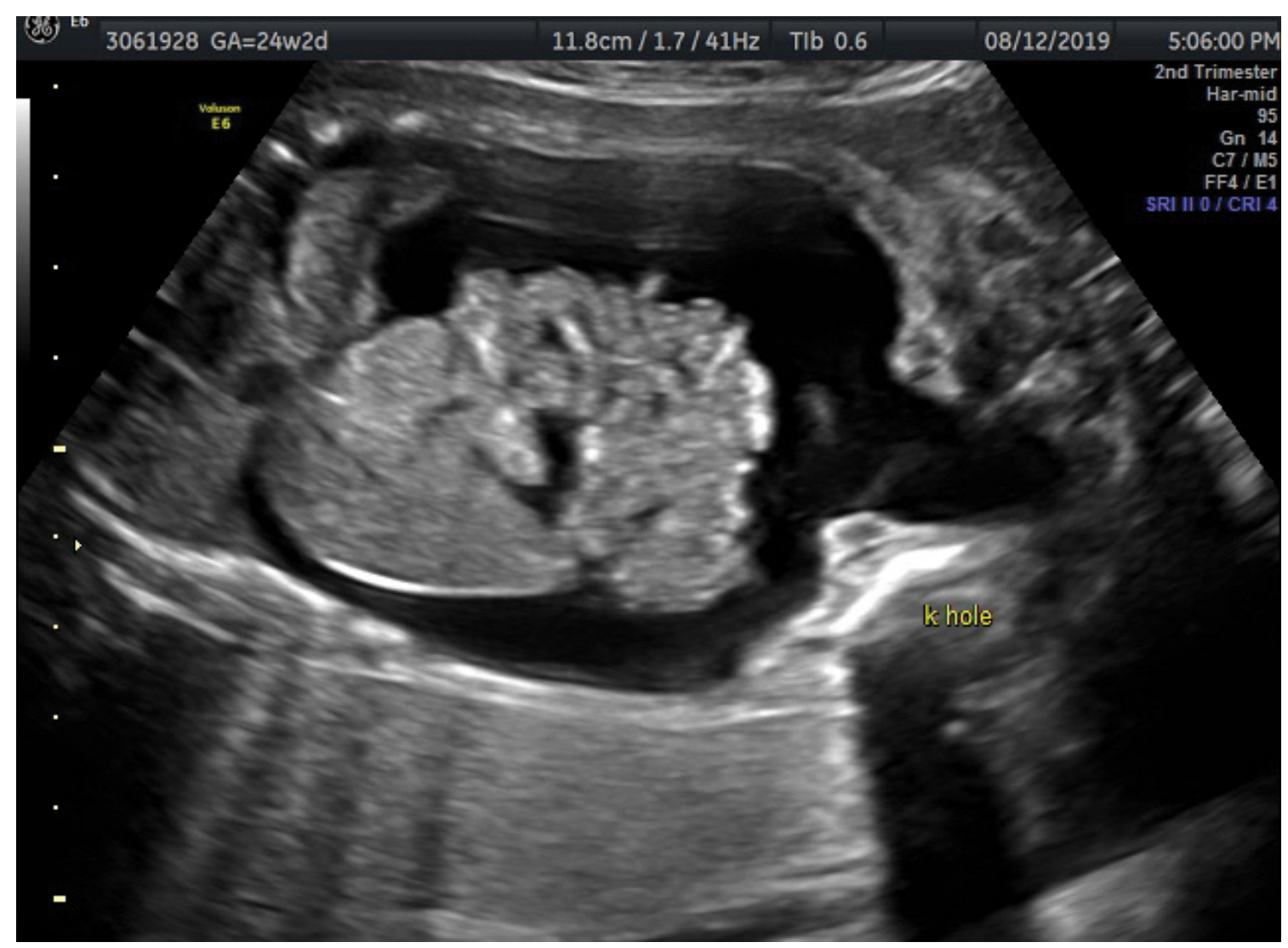

Figure 3: Keyhole appearance of bladder.

mal. The calculated gestational age corresponded to the date of her amenorrhea. Nuchal translucency in the first trimester was normal, and second trimester non-invasive prenatal screening was low risk. Amniocentesis was performed the following day, karyotype, microarray, Noonan syndrome panel testing was negative, and TORCH titers were negative; the fetal gender was male. Fetal MRI was performed at 24.6 weeks, which revealed oligohydramnios and suspected pulmonary hypoplasia. The patient was counseled on possible outcomes, including intrauterine fetal demise, multiple anomalies, and poor long-term prognosis. The patient opted to proceed with all life preserving therapeutic interventions.

At 25.6 weeks gestation, an intrauterine paracentesis of ascites fluid was performed as both a diagnostic and therapeutic procedure. From the fetal abdomen, a $210 \mathrm{cc}$ of yellow ascites fluid was aspirated, and $60 \mathrm{cc}$ was sent for cell count and culture. Additionally, $60 \mathrm{cc}$ of room temperature, normal saline was infused into the amniotic cavity. Cultured ascites fluid was found to be consistent with amniotic fluid. Fetal swallowing of amniotic fluid was observed on ultrasound. At 26.3 weeks, both ascites and oligohydramnios returned. The leading hypothesis was an intestinal perforation leaking amniotic fluid. At 26.5 weeks, a double pigtail catheter was placed to serve as a peritoneal amniotic shunt. At the same time, a second amnioinfusion was performed, and $450 \mathrm{cc}$ of normal saline was again infused into the amniotic cavity. Over the following two weeks, ascites and AFI gradually improved.
At 28.2 weeks, the fetal urinary bladder appeared grossly over distended, and revealed a distinct "Keyhole" sign (Figure 3). Bilateral hydronephrosis was also observed, indicating a lower urinary tract obstruction (LUTO). AFI appeared low-normal levels without evidence of oligohydramnios. At 28.5 weeks, hydronephrosis was more pronounced, and a vesicocintesis was performed to further asses the kidney function. From the fetal bladder, $120 \mathrm{cc}$ of urine was removed; a third amnioinfusion was completed using 250 cc of normal saline. Fetal urine was sent for biochemistry and resulted with elevated levels of beta 2 microglobulin; urine osmolality and sodium levels were normal. With a clearer clinical picture of a lower urinary tract obstruction, the placement of a vesico amniotic shunt was considered. Ultimately not pursued as AFI had remained stable with no sign of recurring oligohydramnios over the following weeks. Betamethasone was administered surrounding the multiple procedures.

Twice weekly fetal surveillance revealed development of bilateral hydronephrosis, much more pronounced on right fetal kidney. Normal blood flow was noted within the left kidney, with decreased perfusion to right kidney (Figure 4). Fetal ascites and distended fetal bladder were also consistently noted. AFI lingered at low-normal limits, around 8-10\%. At 34.5 weeks, a sonogram revealed a sudden drop in AFI (2\%), in conjunction with resolving fetal ascites and smaller appearing bladder; all signs pointed to decreased amniotic fluid production and pending renal failure. Baby was delivered 


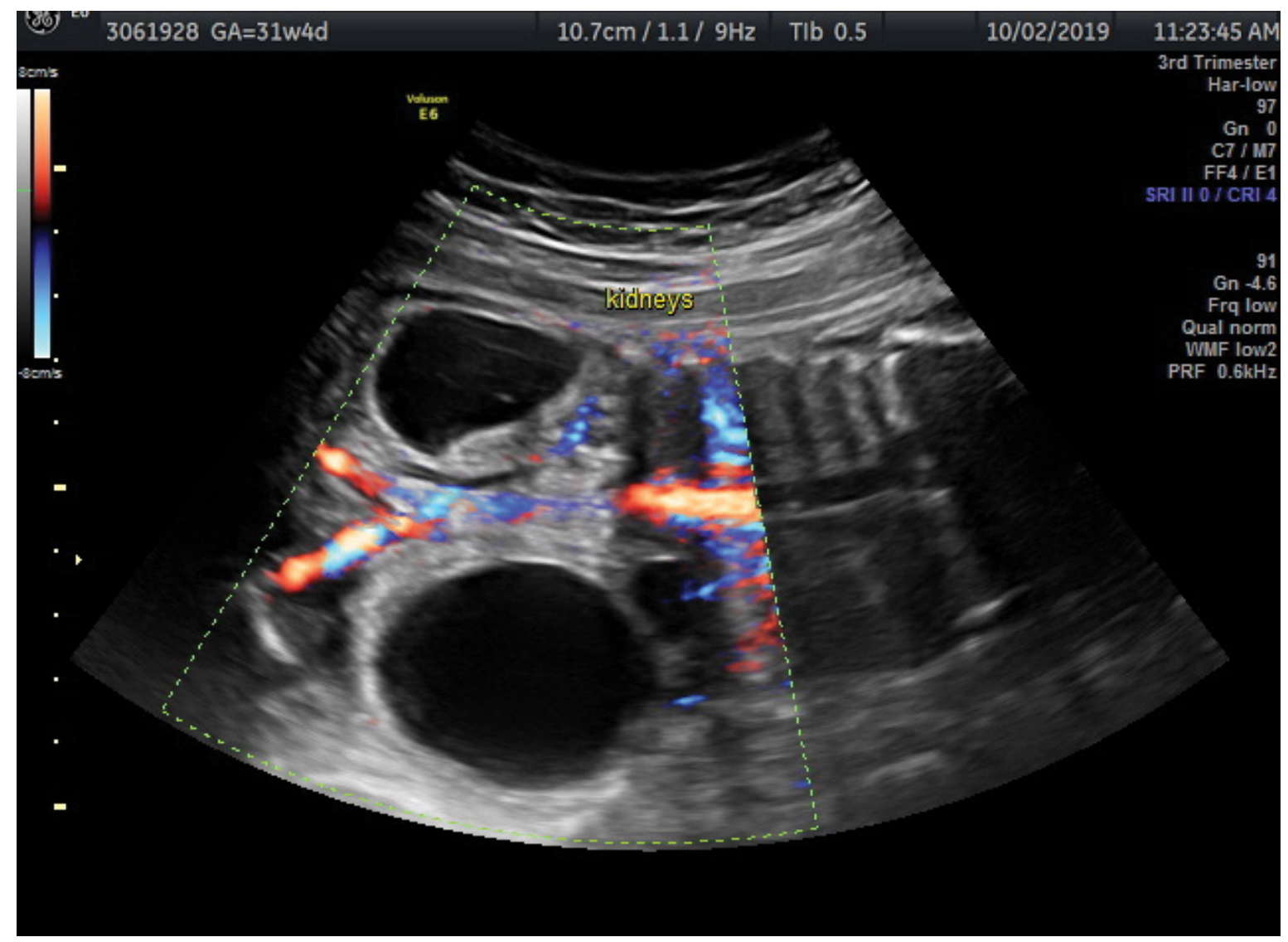

Figure 4: Right fetal kidney.

via emergent cesarean section at 34.5 weeks gestation, weighing 2070 grams. APGARS were 8 and 9 at 1 and 5 minutes. Baby required respiratory support at birth due to increased work of breathing. At 21 days of life, baby was weaned off the respirator and was stable on room air. Renal US showed cystic dysplasia in the setting of posterior urethral valves (PUV). Diagnosis of PUV was confirmed by voiding cystourethrogram (VCUG). Fetal serum creatinine was trending up since birth, started at 0.80 and reached 3.53 by day 4 of life. Posterior urethral valve ablation was performed on day 4 . The degree of cystic dysplasia and echogenicity of kidneys appeared improved on sonogram, 1 week following procedure. Baby was discharged home in stable condition at 8 weeks, with a stable serum creatinine of 0.46 .

This was a spontaneously conceived pregnancy. Throughout the pregnancy the patient reported active fetal movement, and was without any contractions, leakage of fluid, or vaginal bleeding. There were no hospitalizations or major illnesses during the pregnancy. There was no known exposure to any teratogens or ionizing radiation. Her past gynecologic history is significant for polycystic ovarian syndrome. Past medical history is significant for hypothyroidism; diagnosed this pregnancy. Her family history is noncontributory. Medications throughout her pregnancy include Synthroid, prenatal vitamins, omega-3 and probiotics. Her past surgical history is unremarkable. She does not have any allergies or psychological history. She is a home maker, and denied any tobacco, alcohol, and drug use.

\section{Discussion}

We present this interesting case of PUV as it was unique in its atypical initial ultrasound findings of fetal ascites and scalp edema, as most cases of PUV present with increased bladder wall thickness, bladder distension, and hydronephrosis [1,2]. The majority of cases reported in the literature occur sporadically; however, there have been cases associated with autosomal recessive, $x$-linked recessive and polygenic or multifactorial inheritance [3-5]. A full genetic workup was normal, and the patient elected all minimally invasive procedures available to keep the fetus alive.

Early oligohydramnios carries a poor prognosis with the highest risk for pulmonary hypoplasia, renal dysplasia, limb contractures, and a mortality rate of $80-90 \%$ [6-8]. Management guidelines and patient selection for fetal intervention are not well defined. Amnioinfusion is a relatively recent procedure which offers improved chance of survival and does not appear to induce greater complications than conservative management for oligohydramnios [9]. A systemic review was performed by Warring, et al. on serial amnioinfusion therapy, in cases of oligohydramnios diagnosed at early gestational age secondary to LUTO or chronic renal anomalies. The review concluded serial amnioinfusion therapy can reduce the risk of newborn mortality due to pulmonary compromise [10]. 
The fetus in this case presented with non-immune hydrops fetalis, with significant ascites fluid and scalp edema. If the ascites fluid moved the diaphragm upwards and compressed the lungs, pulmonary hypoplasia leading to respiratory distress could have been fetal. Perinatal maneuvers can be performed to improve fetal hemodynamic status and prolong the pregnancy to support antenatal lung development [11]. Abdominal paracentesis can be performed to decompress the fetal abdomen; however, as seen in this case fluid can re-accumulate quickly. Seeds, et al. were the first to report abdominal peritoneal shunting as useful in managing fetal ascites to avoid recurrent paracentesis [11].

The patient had been seen by four prominent maternal fetal medicine physicians for consultations for persistent oligohydramnios and fetal ascites, without a clear diagnosis. The antenatal diagnosis of posterior urethral valves (PUV) was not made until 28.2 weeks, when sonogram findings involving fetal kidneys and bladder were observed, and suggestive of LUTO. In a male fetus, LUTO seen in the second trimester is almost always due to PUV [12]. At 28.2 weeks, ultrasound revealed distended bladder with increased bladder wall thickness and the distinct "Keyhole sign" representing a dilation of the posterior urethra. The Keyhole sign is the classic prenatal ultrasound sign to diagnose PUV, however more recently it has been associated with a high sensitivity (94\%) but low specificity (43\%). Increased bladder wall thickness and bladder dilation are the best diagnostic indicators for PUV [2]. In this case, all three findings were present. The incidence of PUV in males is estimated at 1:5000 - 1:8000 and is the most common cause of male obstructive uropathy [13].

The decision to perform fetal intervention in the setting of PUV is challenging, and it is typically reserved for fetuses with the most severe forms of obstructive uropathy. Fetal mortality rate due to intervention is estimated to be $43 \%$, with options for intervention, including placement of vesico amniotic catheter, creation of cutaneous urostomy, fetal bladder marsupilization, and fetal endoscopic ablation of valves [14]. Long term implications of fetal intervention for obstructive uropathies remain relatively unknown [15]. In this case, fetal intervention was not pursued, given the fetus was clearly producing urine and obstruction was incomplete.

\section{Conclusion}

This case illustrates an unusual presentation of PUV which was not evident until 28 weeks, however through multiple amnioinfusions and shunt placement, a pregnancy that otherwise would have been terminated was saved to a now normal, happy healthy baby. Amnioinfusion in selected cases and after proper work up and counseling might save some pregnancies.

\section{References}

1. Brownlee E, Wragg R, Robb A, Chandran $H$, Knight $M$, et al. (2019) Current epidemiology and antenatal presentation of posterior urethral valves: Outcome of BAPS CASS National Audit. J Pediatr Surg 54: 318-321.

2. Bernardes LS, Aksnes G, Saada J, V Masse, C Elie, et al. (2009) Keyhole sign: How specific is it for the diagnosis of posterior urethral valves? Ultrasound Obstet Gynecol 34: 419-423.

3. Trembath DG, Rijhsinghani A (2002) Possible maternal inheritance of a common obstructive urinary tract anomaly. Report of a case of a woman with multiple urinary tract infections and two sons with posterior urethral valves. J Reprod Med 47: 962-964.

4. Schreuder MF, van der Horst HJR, Bokenkamp A, Beckers GMA, van Wijk JAE (2008) Posterior urethral valves in three siblings: A case report and review of the literature. Birth Defects Res A Clin Mol Teratol 82: 232-235.

5. Satish Kumar K, Venkatesh (2017) The familial occurrence of posterior urethral valve: A possible clue towards genetic etiology. EC Paediatrics 6: 60-66.

6. Barss VA, Benacerraf BR, Frigoletto FD (1984) Second trimester oligohydramnios, a predictor of poor fetal outcome. Obstet Gynecol 64: 608-610.

7. Mercer LJ, Brown LG (1986) Fetal outcome with oligohydramnios in the second trimester. Obstetrics and Gynecology 67: $840-842$.

8. Christianson C, Huff D, McPherson E (1999) Limb deformations in oligohydramnios sequence: Effects of gestational age and duration of oligohydramnios. Am J Med Genet 86: 430-433.

9. Gramellini D, Fieni S, Kaihura C, Faiola S, Vadora E (2003) Transabdominal antepartum amnioinfusion. International Journal of Gynecology \& Obstetrics 83: 171-178.

10. Warring SK, Novoa V, Shazly S, Mari Charisse Trinidad, David J Sas, et al. (2020) Serial amnioinfusion as regenerative therapy for pulmonary hypoplasia in fetuses with intrauterine renal failure or severe renal anomalies: Systematic review and future perspectives. Mayo Clin Proc Innov Qual Outcomes 4: 391-409.

11. Seeds JW, Herbert WN, Bowes WA, Cefalo RC (1984) Recurrent idiopathic fetal hydrops: Results of prenatal therapy. Obstet Gynecol 64: 30S-33S.

12. Panicker R, Grewal D (2010) Antenatally diagnosed posterior urethral valves: A dilemma. Medical Journal Armed Forces India 66: 167-169.

13. Tambo FFM, Tolefac PN, Ngowe MN, Jacqueline Z, Landry $M$, et al. (2018) Posterior urethral valves: 10 years audit of epidemiologic, diagnostic and therapeutic aspects in Yaounde gynaeco-obstetric and paediatric hospital. BMC Urol 18.

14. Holmes N, Harrison MR, Baskin LS (2001) Fetal surgery for posterior urethral valves: Long-term postnatal outcomes. PEDIATRICS 108: e7-e7.

15. Freedman AL, Johnson MP, Smith CA, Gonzalez R, Evans MI (1999) Long-term outcome in children after antenatal intervention for obstructive uropathies. Lancet 354: 374-377. 